KIAS-P07008

hep-th/0703015

\title{
Meta-Stable Brane Configuration and Gauged Flavor Symmetry
}

\author{
Changhyun Ahn \\ Department of Physics, Kyungpook National University, Taegu 702-701, Korea \\ ahn@knu.ac.kr
}

\begin{abstract}
Starting from an $\mathcal{N}=1$ supersymmetric electric gauge theory with the gauge group $S p\left(N_{c}\right) \times S O\left(2 N_{c}^{\prime}\right)$ with fundamentals for the first gauge group factor and a bifundamental, we apply Seiberg dual to the symplectic gauge group only and arrive at the $\mathcal{N}=1$ supersymmetric dual magnetic gauge theory with dual matters including the gauge singlets and superpotential. By analyzing the F-term equations of the dual magnetic superpotential, we describe the intersecting brane configuration of type IIA string theory corresponding to the meta-stable nonsupersymmetric vacua of this gauge theory.
\end{abstract}




\section{Introduction}

The $\mathcal{N}=1 S U\left(N_{c}\right)$ gauge theory with $N_{f}$ fundamental flavors has a vanishing superpotential before we deform this theory by mass term for quarks. When we add an adjoint field to this theory, then there exists a nonzero superpotential for this adjoint field. On the other hand, when we add a symmetric tensor flavor(or antisymmetric tensor flavor) to $\mathcal{N}=1 S U\left(N_{c}\right)$ SQCD with $N_{f}$ fundamental flavors, in general, there exists a nonzero quartic superpotential consisting of these matter fields. In the type IIA brane configuration, this gauge theory can be described by three NS5-branes, D6-branes, D4-branes and an orientifold 6-plane. The coefficient functions appearing the above quartic superpotential depend on both how the two outer NS5-branes are rotated with respect to a middle NS5-brane and how the D6-branes are rotated with respect to a middle NS5-brane. Then by tuning these two rotation angles in appropriate way, one can make the above nonzero quartic superpotential to vanish. For the brane configuration description corresponding to $\mathcal{N}=1$ supersymmetric gauge theory, see the review paper [1].

Both deforming the electric gauge theory by adding the mass for the quarks and taking the Seiberg dual magnetic theory from the electric theory are necessary to find out meta-stable supersymmetry breaking vacua in the context of dynamical supersymmetry breaking [2, 3]. When we take the Seiberg dual from the electric theory, the vanishing superpotential in the electric theory makes it easier to deal with its nonvanishing dual magnetic superpotential since the reduced superpotential in the magnetic theory has simple form to analyze.

On the other hand, the $\mathcal{N}=1$ product gauge group $S U\left(N_{c}\right) \times S U\left(N_{c}^{\prime}\right)$ with $N_{f}$ fundamentals, $N_{f}^{\prime}$ fundamentals and two bifundamentals can be described by similar expression for the superpotential to the one in the single gauge group in previous paragraph. In general, one can also think of a multiple NS5-branes for the outer NS5-branes together with a single middle NS5-brane. Since there is no orientifold 6-plane in this case, compared with previous brane configuration, the rotations of outer NS5-branes and those of D6-branes with respect to a middle NS5-brane are completely arbitrary. Therefore, the coefficient functions in the superpotential depend on four rotation angles rather than two. Then by tuning these four rotation angles appropriately, one can make the nonzero superpotential to vanish in the electric theory.

Let us add an orientifold 4-plane in this brane configuration for product gauge group with matter contents we mentioned in previous paragraph. One expects that the gauge group will be a product gauge group between a symplectic group with $O 4^{+}$-plane and an orthogonal group with $\mathrm{O4}^{-}$-plane. Under this orientifolding, the matter contents should be 
changed appropriately: one kind of fundamental flavors for each gauge group factor and one bifundamental. This particular brane configuration with $O 4^{ \pm}$-planes will play the important role in this paper: three NS5-branes, D6-branes, D4-branes and an orientifold 4-plane.

A non-chiral model of dynamical supersymmetry breaking with stable nonsupersymmetric vacua was found in [4, 5] 1. By gauging the "maximal" subgroup of the flavor symmetry group of this model, the existence of runaway behavior on the scalar potential at the region of the number of flavors corresponding to [4, 5] was explained by using the brane configuration [6] in the magnetic theory where there are no D6-branes. Moreover, in [7], the different gauging of subgroup of the flavor symmetry group 2 leads to two facts: for the nonabelian gauged flavor group, the M5-brane configuration with massless quarks is nonholomorphic while for the $U(1)$ gauged flavor group, supersymmetry breaking with stable nonsupersymmetric vacua with massless quarks involving $t$-configuration exists by analyzing the corresponding holomorphic M5-brane configuration.

In this paper, starting from an $\mathcal{N}=1$ supersymmetric electric gauge theory, we obtain the $\mathcal{N}=1$ supersymmetric dual magnetic gauge theory(with dual matters and corresponding superpotential) which is the model of [4, 5] with "gauged" flavor symmetry group. Based on this magnetic brane configuration, we describe the intersecting brane configuration of type IIA string theory corresponding to the meta-stable nonsupersymmetric vacua of this gauge theory by recombination of color D4-branes and flavor D4-branes and moving those D4-branes to $(45)$ directions.

In section 2, we review the type IIA brane configuration corresponding to the electric theory based on the $\mathcal{N}=1 S p\left(N_{c}\right) \times S O\left(2 N_{c}^{\prime}\right)$ gauge theory with matter contents and deform this theory by adding the mass term for the quarks. Then we construct the Seiberg dual magnetic theory which is $\mathcal{N}=1 S p\left(\widetilde{N}_{c}\right) \times S O\left(2 N_{c}^{\prime}\right)$ gauge theory with corresponding dual matters as well as various gauge singlets, by brane motion and linking number counting.

In section 3, we consider the nonsupersymmetric meta-stable minimum by looking at the magnetic brane configuration we obtained in section 2 and present the corresponding intersecting brane configuration of type IIA string theory, along the line of [10, 11, 12, 13, 14, 15, 16] and we describe M-theory lift of this supersymmetry breaking type IIA brane configuration.

In section 4 , we make some comments on the future directions.

\footnotetext{
${ }^{1}$ It is called by ITIY model or IYIT model.

${ }^{2}$ In [8], the diagonal flavor group of $\mathcal{N}=1 S U\left(N_{c}\right)$ SQCD with massive flavors is gauged and the additional color singlets are inserted both in an electric theory and magnetic theory. It is an open problem how to realize this gauge theory from the type IIA brane configuration. On the other hand, in 9, the flavor group is gauged but the Seiberg dual is taken on this gauged flavor group rather than color group.
} 


\section{The $\mathcal{N}=1$ supersymmetric electric and magnetic brane configuration}

In this section, we consider $\mathcal{N}=1 S p\left(N_{c}\right)$ gauge theory with gauged flavor symmetry group $S O\left(2 N_{c}^{\prime}\right)$ in electric theory first and then we move on its dual magnetic gauge theory $\mathcal{N}=1$ $S p\left(\widetilde{N}_{c}\right)$ gauge theory with the same gauged flavor symmetry group $S O\left(2 N_{c}^{\prime}\right)$. We present the corresponding brane configurations in Figure 1 and Figure 2 respectively.

\subsection{Electric theory}

The gauge group of an electric theory [7] is given by $S p\left(N_{c}\right) \times S O\left(2 N_{c}^{\prime}\right)$ and the matter contents are 3

- $2 N_{f}$ quark fields $Q$ is in the representation $\left(\mathbf{2 N}_{\mathbf{c}}, \mathbf{1}\right)$ under the gauge group

- The flavor-singlet field $X$ is in the bifundamental representation $\left(\mathbf{2} \mathbf{N}_{\mathbf{c}}, \mathbf{2} \mathbf{N}_{\mathbf{c}}^{\prime}\right)$ under the gauge group

The anomaly free global symmetry is given by $S U\left(2 N_{f}\right) \times U(1)_{R}$ and the strong coupling scales for $S p\left(N_{c}\right)$ and $S O\left(2 N_{c}^{\prime}\right)$ are denoted by $\Lambda_{1}$ and $\Lambda_{2}$ respectively. The theory is asymptotically free when $3\left(2 N_{c}+2\right)-2 N_{f}-2 N_{c}^{\prime}>0$ for the $S p\left(N_{c}\right)$ gauge theory and when $3\left(2 N_{c}^{\prime}-2\right)-2 N_{c}>0$ for the $S O\left(2 N_{c}^{\prime}\right)$ gauge theory by computing the coefficients of beta function.

The type IIA brane configuration for this theory [7] can be described by $2 N_{c}$ color D4branes (01236) suspended between a middle NS5-brane (012345) and the right NS5'-brane (012389) along $x^{6}$ direction together with $2 N_{f}$ D6-branes(including mirrors) at values of $x^{6}$ that are between those corresponding to the positions of the middle NS5-brane and the right NS5'-brane. Moreover, an extra the left NS5'-brane is located at the left hand side of a middle NS5-brane along the $x^{6}$ direction and there exist $2 N_{c}^{\prime}$ color D4-branes suspended between them. Of course, there is an orientifold 4-plane (01236) which acts as $\left(x^{4}, x^{5}, x^{7}, x^{8}, x^{9}\right) \rightarrow$ $\left(-x^{4},-x^{5},-x^{7},-x^{8},-x^{9}\right)$. The RR charge of O4-plane flips sign each time it passes through NS5-brane or NS5'-brane [19]. These are shown in Figure 1.

The classical superpotential by deforming this theory by adding the mass term for the

\footnotetext{
${ }^{3}$ By introducing the chiral multiplets $X_{1}$ and $X_{2}$ that are symmetric tensor in $S p\left(N_{c}\right)$ and antisymmetric tensor in $S O\left(2 N_{c}^{\prime}\right)$ respectively, the $S p\left(N_{c}\right) \times S O\left(2 N_{c}^{\prime}\right)$ gauge theory in different context can be described by the tree-level superpotential that is given by $W=W_{1}\left(X_{1}\right)+W_{2}\left(X_{2}\right)+Q_{12}^{T} X_{1} Q_{12}+Q_{12} X_{2} Q_{12}^{T}$ where $Q_{12}$ is a bi-fundamental and $W_{i}\left(X_{i}\right)$ is a polynomial for the field $X_{i}$. See also the same gauge theory with different matters in [17. where there exist more meson fields in the magnetic theory and see [18 for unitary gauge group in the quiver gauge theory.
} 
quarks is given by 4

$$
W=m Q Q
$$

and this brane configuration can be summarized as follows 5 :

- One middle NS5-brane(012345)

- Two NS5'-branes(012389)

- $2 N_{f}$ D6-branes(0123789)

- $2 N_{c}$ color D4-branes(01236)

- $2 N_{c}^{\prime}$ color D4-branes(01236)

- One O4-plane(01236)

Now we draw this electric brane configuration in Figure 1 which was already found in [7] for the massless quarks and we put the coincident D6-branes in the nonzero $v$ direction(and its mirrors). The electric quarks $Q$ correspond to $4-4$ strings connecting the $N_{c}$ color D4-branes with $N_{f}$ flavor D4-branes(and its mirrors) where one can see these flavor D4-branes more clearly if move $N_{f}$ D6-branes to the left all the way past NS5-brane(and its mirrors) and after that there exist newly created $N_{f}$ D4-branes connecting $N_{f}$ D6-branes and NS5-brane in this process. The bifundamentals $X$ correspond to 4 -4 strings connecting the $N_{c}$ color D4-branes with $N_{c}^{\prime}$ color D4-branes(and its mirrors) 6 .

\subsection{Magnetic theory}

Let us take the Seiberg dual for the first gauge group factor $\operatorname{Sp}\left(N_{c}\right)$ while keeping the second gauge group factor $S O\left(2 N_{c}^{\prime}\right)$ untouched. Suppose that $\Lambda_{1}>>\Lambda_{2}$. This can be done by field theory side or type IIA string theory side via brane motion. Let us do this by latter [25, 26, 1, 14, 13]. After we move a middle NS5-brane to the right all the way past the

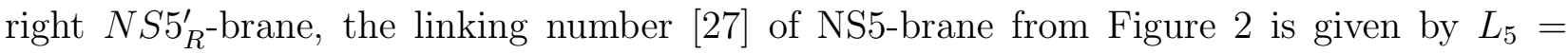
$\frac{\left(2 N_{f}\right)}{2}-1-(1)-2 \widetilde{N}_{c}$. Note that $O 4^{+}$-plane with $\mathrm{RR}$ charge +1 realizes a symplectic gauge

\footnotetext{
${ }^{4}$ The mass matrix $m$ in (2.1) is antisymmetric in the flavor indices and is given by $-i \sigma^{2} \otimes$ $\operatorname{diag}\left(m_{1}, m_{2}, \cdots, m_{N_{f}}\right)$ where $\sigma^{2}$ is a second Pauli matrix explicitly. The quadratic term in (2.1) is given by $Q J Q$ explicitly where the color indices are contracted. Here $J$ is a symplectic metric and antisymmetric in the color indices and is given by $J=i \sigma^{2} \otimes \mathbf{1}_{N_{c} \times N_{c}}$ explicitly.

${ }^{5}$ We introduce two complex coordinates $v \equiv x^{4}+i x^{5}$ and $w \equiv x^{8}+i x^{9}$, as usual.

${ }^{6}$ This brane configuration can be obtained from the brane configuration corresponding to the product gauge group $S U\left(N_{c}\right) \times S U\left(N_{c}^{\prime}\right)$ theory [20, 21] with different matter contents. The superpotential, in general, has the form of $W=m_{1} X_{1}^{2}+m_{2} X_{2}^{2}+X_{1} \widetilde{F} F+X_{2} \widetilde{F} F+Q X_{1} \widetilde{Q}+Q^{\prime} X_{2} \widetilde{Q}^{\prime}$. The masses are given by $m_{1}=\tan \theta_{1}$ and $m_{2}=\tan \theta_{2}$ where $\theta_{i}$ is an rotation angle [22] of outer $N S 5_{\theta_{i}}$-brane with respect to a middle NS5-brane. This superpotential can be reduced after O4-plane orientifolding procedure 23, 24]. By integrating the adjoint fields $X_{1}$ and $X_{2}$, the superpotential is given by $W \sim\left(\frac{1}{m_{1}}+\frac{1}{m_{2}}\right) X^{4}$. Now it is evident that for $\theta_{1}=\theta_{2}=\frac{\pi}{2}$, the superpotential vanishes as we observed above.
} 


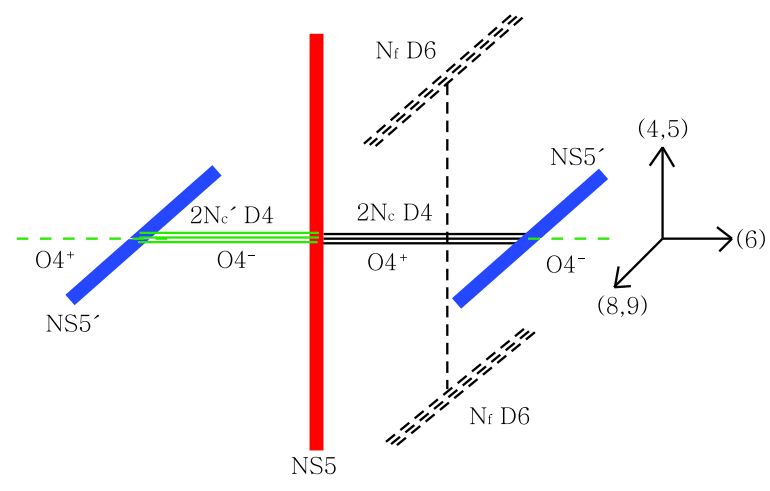

Figure 1: The $\mathcal{N}=1$ supersymmetric electric brane configuration for the $S p\left(N_{c}\right) \times S O\left(2 N_{c}^{\prime}\right)$ gauge theory with $2 N_{f}$ quark fields $Q$ in the fundamental representation and singlet field $X$ in the bifundamental representation. The origin of $(v, w)$ is located at the intersection between NS5-brane and D4-branes. The D6-branes have nonzero $v$ coordinates where $v= \pm m$ for equal massive case.

group while $O 4^{-}$-plane with $\mathrm{RR}$ charge -1 does an orthogonal gauge group. So we put $n_{4 R}=-1$ due to the $O 4^{-}$-plane and $n_{4 L}=1$ due to the $O 4^{+}$-plane above as well as the contributions from the D4-branes. Originally, it was $L_{5}=-\frac{\left(2 N_{f}\right)}{2}+1-(-1)+2 N_{c}-2 N_{c}^{\prime}$ from Figure 1 before the brane motion. Also in this case, we put $n_{4 R}=1$ due to the $O 4^{+}$-plane and $n_{4 L}=-1$ due to the $O 4^{-}$-plane. Therefore, by the linking number conservation and equating these two $L_{5}$ 's each other, we are left with the number of colors in the magnetic theory [7]

$$
\widetilde{N}_{c}=N_{f}+N_{c}^{\prime}-N_{c}-2 .
$$

Let us draw this magnetic brane configuration in Figure 2 which was already found in [7] for the massless quarks and we put the coincident D6-branes in the nonzero $v$ directions(and its mirrors). During this process from an electric theory to the magnetic theory, the $N_{f}$ created D4-branes(and its mirrors) connecting between D6-branes and NS5'-brane can move freely in the $w$ direction. Moreover since $2 N_{c}^{\prime}$ D4-branes are suspending between two equal $N S 5_{L, R^{-}}^{\prime}$ branes located at different $x^{6}$ coordinate, these $\mathrm{D} 4$-branes can slide along the $w$ direction also.

Then the weakly coupled dual magnetic gauge group 7 at the scale $E$ where $\Lambda_{2}<<E$ is given by $S p\left(\widetilde{N}_{c}\right) \times S O\left(2 N_{c}^{\prime}\right)$ and the matter contents are

${ }^{7}$ The $\mathcal{N}=1 S p\left(\widetilde{N}_{c}\right)$ gauge theory with matter contents $\widetilde{Q}$ and $S$ and the superpotential $W_{\text {dual }}=S \widetilde{Q} \widetilde{Q}+m S$ can be interpreted as a dual magnetic description of $S p\left(N_{c}=N_{f}-\widetilde{N}_{c}-2\right)$ gauge theory with $2 N_{f}$ massive quarks when the number of flavors satisfies $N_{f}>\widetilde{N}_{c}+2$. Then the Intriligator-Thomas-Izawa-Yanagida model(which can be also denoted as IYIT model in some literatures) [28, 29, 4, 5] can be obtained as an electric $\operatorname{Sp}\left(N_{c}=-1\right)$ gauge theory with the condition $N_{f}=\widetilde{N}_{c}+1$ [7]. For this particular number of flavor, there exists a quantum constraint $\operatorname{Pf} S=\widetilde{\Lambda}^{2 N_{f}}$ [30] and this leads to the supersymmetric vacua when $m^{N_{f}}=\widetilde{\Lambda}^{2 N_{f}}$ [6, 31] which corresponds to a supersymmetric M5-brane configuration. 


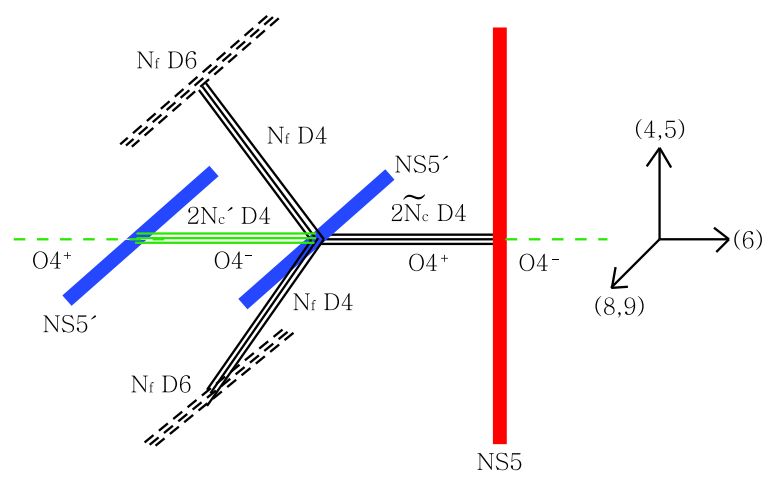

Figure 2: The $\mathcal{N}=1$ supersymmetric magnetic brane configuration for the $\operatorname{Sp}\left(\widetilde{N}_{c}=N_{f}+\right.$ $\left.N_{c}^{\prime}-N_{c}-2\right) \times S O\left(2 N_{c}^{\prime}\right)$ gauge theory with $2 N_{f}$ quark fields $\widetilde{Q}$, a singlet field $\widetilde{X}$, the gauge singlet $\Phi, 2 N_{f}$ fields $N$, and an antisymmetric two index tensor $S$.

- $2 N_{f}$ quark fields $\widetilde{Q}$ in the $\left(\mathbf{2} \widetilde{\mathbf{N}}_{\mathbf{c}}, \mathbf{1}\right)$

- the flavor-singlet field $\widetilde{X}$ in the bifundamental representation $\left(\mathbf{2} \widetilde{\mathbf{N}}_{\mathrm{c}}, \mathbf{2} \mathbf{N}_{\mathrm{c}}^{\prime}\right)$

- the gauge-singlet $\Phi$ in the representation $\left(\mathbf{1}, \mathbf{N}_{\mathbf{c}}^{\prime}\left(2 \mathbf{N}_{\mathbf{c}}^{\prime}-\mathbf{1}\right)\right)$

- $2 N_{f}$ fields $N$ in $\left(\mathbf{1}, \mathbf{2} \mathbf{N}_{\mathbf{c}}^{\prime}\right)$

- an antisymmetric two index tensor(in the flavor indices) $S$ in the representation $(\mathbf{1}, \mathbf{1})$

Magnetic quarks $\widetilde{Q}$ correspond to 4-4 strings connecting the $\widetilde{N}_{c}$ color D4-branes with $N_{f}$ flavor D4-branes(and its mirrors), $\widetilde{X}$ corresponds to to 4-4 strings connecting the $\widetilde{N}_{c}$ color D4-branes with $N_{c}^{\prime}$ color D4-branes(and its mirrors), $\Phi$ is a flavor-singlet corresponding to 4-4 strings connecting two different color D4-branes among $N_{c}^{\prime}$ color D4-branes(and its mirrors), $N$ corresponds to 4-4 strings connecting the $N_{c}^{\prime}$ color D4-branes with $N_{f}$ flavor D4-branes(and its mirrors) and $S$ corresponds to 4-4 strings connecting two different flavor D4-branes among $N_{f}$ flavor D4-branes(and its mirrors).

In the dual theory, since there exist $2 N_{f}$ fundamental fields $\widetilde{Q}$ and one bifundamental field $\widetilde{X}$ which will give rise to the contribution of $2 N_{c}^{\prime}$, the coefficient of the beta function 8 is $b_{S p\left(\widetilde{N}_{c}\right)}=3\left(2 \widetilde{N}_{c}+2\right)-2 N_{f}-2 N_{c}^{\prime}$ and since there are $2 \widetilde{N}_{c}$ fundamental fields $\widetilde{X}$ for the flavor index of the first factor, an antisymmetric tensor $\widetilde{\Phi}$ which will contribute to $\left(2 N_{c}^{\prime}-2\right)$ and $2 N_{f}$ fields $N$, the coefficient of the beta function is $b_{S O\left(2 N_{c}^{\prime}\right)}=3\left(2 N_{c}^{\prime}-2\right)-2 \widetilde{N}_{c}-\left(2 N_{c}^{\prime}-2\right)-2 N_{f}$. Therefore, both $S p\left(\widetilde{N}_{c}\right)$ and $S O\left(2 N_{c}^{\prime}\right)$ gauge couplings are IR free by requiring the negativeness of the coefficients of beta function. One can rely on the perturbative calculations at low energy for this magnetic IR free region $b_{S p\left(\widetilde{N}_{c}\right)}<0$ and $b_{S O\left(2 N_{c}^{\prime}\right)}<0$.

The dual magnetic tree level superpotential, by adding the mass term for the $Q$ in electric

${ }^{8}$ The index of representation $R$ is given by $K\left(N_{1}\right)=2, K($ symm. $)=2\left(N_{1}+2\right)$ and $K($ adj $=$ antisymm. $)=$ $2\left(N_{1}-2\right)$ for $S O\left(N_{1}\right)$ group and $K\left(2 N_{2}\right)=2, K($ antisymm. $)=2\left(2 N_{2}-2\right)$ and $K($ adj. $=$ symm. $)=2\left(2 N_{2}+2\right)$ for $S p\left(N_{2}\right)$ group. Recall that the coefficient of the beta function is $b=\frac{1}{2}\left[3 K(\right.$ adj. $)-\sum$ matter $\left.K(R)\right]$. 
theory corresponding to add a linear term in $S$ in dual magnetic theory [7], is given by

$$
W_{\text {dual }}=(S \widetilde{Q} \widetilde{Q}+\widetilde{X} \Phi \widetilde{X}+\widetilde{Q} N \widetilde{X})+m S
$$

where one can add intermediate scale in the meson terms relating the strong coupling scale $\Lambda_{1}$ of the electric gauge group $S p\left(N_{c}\right)$ to the scale of $\widetilde{\Lambda}_{1}$ of its magnetic dual gauge group $\operatorname{Sp}\left(\widetilde{N}_{c}\right)$. Here the mesons are given, in terms of the fields defined in the electric theory, by 9

$$
S \equiv Q Q, \quad \Phi \equiv X X, \quad N \equiv Q X
$$

The fluctuations of the singlet $S$ correspond to the motion in the (789) directions of the flavor D4-branes in Figure 2. Similarly, the fluctuations of the singlet $\Phi$ correspond to the motion in the (789) directions of the D4-branes suspended two NS5'-branes in Figure 2. Here $\widetilde{Q}$ is fundamental for the gauge group index and antifundamental for the flavor index. Then, $\widetilde{Q} \widetilde{Q}$ has rank $2 \widetilde{N}_{c}$ while $m$ has a rank $2 N_{f}$. Therefore, the F-term condition, the derivative the superpotential $W_{\text {dual }}$ with respect to $S$, cannot be satisfied if the rank $2 N_{f}$ exceeds $2 \widetilde{N}_{c}$. This is so-called rank condition and the supersymmetry is broken.

The classical moduli space of vacua can be obtained from F-term equations. From the F-terms $F_{\widetilde{Q}}$ and $F_{S}$, one gets $S \widetilde{Q}+N \widetilde{X}=0=\widetilde{Q} \widetilde{Q}+m$. Similarly, one obtains $\Phi \widetilde{X}+\widetilde{Q} N=$ $0=\widetilde{X} \widetilde{X}$ from the F-terms $F_{\tilde{X}}$ and $F_{\Phi}$. Moreover, there is a relation $\widetilde{Q} \widetilde{X}=0$ from the F-term $F_{N}$. Then, one obtains the following solutions

$$
<\widetilde{Q}>=\left(\begin{array}{c}
i \sqrt{m} \mathbf{1}_{2 \widetilde{N}_{c}} \\
0
\end{array}\right), \quad<S>=\left(\begin{array}{cc}
0 & 0 \\
0 & \Phi_{0} \mathbf{1}_{\left(N_{f}-\widetilde{N}_{c}\right)} \otimes i \sigma^{2}
\end{array}\right), \quad<\widetilde{X}>=0=<N>
$$

where $\Phi_{0} \mathbf{1}_{\left(N_{f}-\widetilde{N}_{c}\right)} \otimes i \sigma^{2}$ is an arbitrary $2\left(N_{f}-\widetilde{N}_{c}\right) \times 2\left(N_{f}-\widetilde{N}_{c}\right)$ antisymmetric matrix and the zeros of $\langle\widetilde{Q}\rangle$ are $2\left(N_{f}-\widetilde{N}_{c}\right) \times 2 \widetilde{N}_{c}$ zero matrices. Similarly, the zeros of $2 N_{f} \times 2 N_{f}$ matrix $S$ are assumed also. Then $\Phi_{0}$ and $i \sqrt{m}$ parametrize a pseudo-moduli space. Let us expand around on a point on (2.3) as done in [2]. That is,

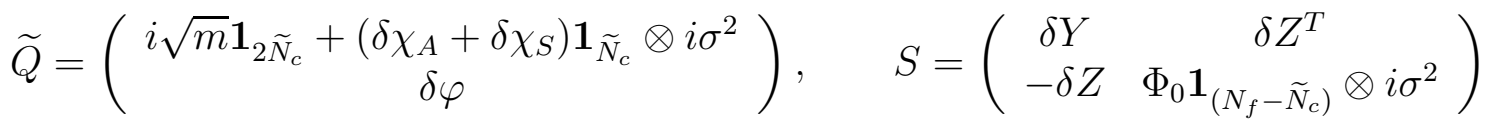

as well as the fluctuations for $N$ and $\widetilde{X}$. Then the superpotential becomes

$$
\begin{aligned}
W_{\text {dual }}^{\text {fluct }} & =\Phi_{0}(\delta \varphi \delta \varphi+m)+\delta Z^{T} \delta \varphi i \sqrt{m}+\delta Z i \sqrt{m} \delta \varphi \\
& +\left(\delta Y \delta \chi_{A} i \sqrt{m}+\cdots\right)+(\text { cubic })
\end{aligned}
$$

${ }^{9}$ The meson field $S$ is given by $Q J Q$ where the color indices are contracted. Here $J$ is given by the footnote 4. Similarly, the meson field $\Phi$ is given by $X J X$ and the meson field $N$ is $Q J X$ where the color indices are contracted. 
where (cubic) stands for the terms that are cubic or higher in the fluctuations and $\cdots$ contains some parts from the second and third terms in (2.2) that are not relvant: There are two kinds of terms, the vacuum of $\langle\widetilde{Q}>$ multiplied by $\delta N \delta \widetilde{X}$ and the vacuum of $\langle\Phi>$ multiplied by $\delta \tilde{X} \delta \widetilde{X}$. By redefining these as $\delta \hat{N} \delta \hat{\widetilde{X}}$ and $\delta \hat{\widetilde{X}} \delta \hat{\widetilde{X}}$ respectively, they do not enter the contributions for the one loop result. Then to quadratic order, the model splits into two sectors where the first piece given by the first line of (2.4) is an O'Raifeartaigh type model and the second piece given by the second line of (2.4) is supersymmetric and will not contribute to the supertrace. The fields $\delta \chi_{A, S}$ and $\delta Y$ couple to the supersymmetry breaking fields $\delta \varphi$ via terms of cubic and higher order in the fluctuations. Then, the remaining relevant terms of superpotential are given by the first line of (2.4). At one loop, the effective potential $V_{e f f}^{(1)}$ for $\Phi_{0}$ can be obtained from this superpotential which consists of the matrices $M$ and $N$ of [32] where the defining function $\mathcal{F}\left(v^{2}\right)$ can be computed. Using the equation (2.14) of [32] of $m_{\Phi_{0}}^{2}$ and $\mathcal{F}\left(v^{2}\right)$, one gets that $m_{\Phi_{0}}^{2}$ will contain $(\log 4-1)>0$. This implies that these vacua are stable.

Note that in the original model of [4, 5], when $N_{f}=\widetilde{N}_{c}+1$, the result of [33] implies that when the extremization of a tree-level superpotential with massless quarks conflicts with a quantum constraint, as observed in the footnote 7 , the low energy effective theory near the origin of moduli space is an O'Raifeartaigh model [34] and the sign of mass squared for the pseudoflat direction at the origin is calculable and there is a local minimum around the origin. In this case the $U(1)_{R}$ symmetry is preserved. On the other hand, the effective theory becomes noncalcuable for large moduli space. For the discussion of $S p(1) \sim S U(2)$ SQCD with two flavors, see [28, 29, 35].

Remember that in the original model of [4, 5], the global flavor group is given by $S U\left(2 \widetilde{N}_{c}+\right.$ 2) and in [7] the $S O\left(2 N_{c}^{\prime}\right)$ subgroup of $S U\left(2 \widetilde{N}_{c}+2\right)$ for massless quarks was gauged where $1 \leq N_{c}^{\prime} \leq \widetilde{N}_{c}$ while the "maximal" subgroup $S O\left(2 \widetilde{N}_{c}+2\right)$ of $S U\left(2 \widetilde{N}_{c}+2\right)$ was gauged in [6]. The above magnetic brane configuration can be also obtained directly from $\mathcal{N}=1 \operatorname{Sp}\left(\tilde{N}_{c}\right)$ SQCD with $N_{f}$ fundamentals(let us focus on the massless case) by replacing $2 N_{c}^{\prime}$ D6-branes with an $N S 5_{L}^{\prime}$-brane(attaching the same number $2 N_{c}^{\prime} \mathrm{D} 4$-branes along the $x^{6}$ direction) and then the remaining $2\left(N_{f}-N_{c}^{\prime}\right)$ D6-branes can be shifted to the new $2 N_{f}$ D6-branes which is consistent with the global symmetry group $S U\left(2 N_{f}\right)$ [7]. The appropriate consideration for the O4-plane should be included. 


\section{Nonsupersymmetric meta-stable brane configuration}

Now we recombine $\widetilde{N}_{c}$ D4-branes among $N_{f}$ D4-branes connecting between D6-branes and $N S 5_{R}^{\prime}$-brane with those connecting between $N S 5_{R}^{\prime}$-brane and NS5-brane(and its mirrors) and push them in $v$ direction from Figure 2 [16, 15, 14, 13]. Of course their mirrors will move to $-v$ direction in a $\mathbf{Z}_{2}$ symmetric manner due to the $O 4^{+}$-plane. After this procedure, there are no color D4-branes between $N S 5_{R}^{\prime}$-brane and NS5-brane. For the flavor D4-branes, we are left with only $\left(N_{f}-\widetilde{N}_{c}\right)$ D4-branes(and its mirrors).

Then the minimal energy supersymmetry breaking brane configuration is shown in Figure 3. If we ignore the left $N S 5_{L^{\prime}}$-brane, $2 N_{c}^{\prime}$ D4-branes and $O 4^{+}$-plane connecting to the left $N S 5_{L}^{\prime}$-brane(detaching these from Figure 3 ), then this brane configuration corresponds to the minimal energy supersymmetry breaking brane configuration for the $\mathcal{N}=1$ SQCD with the magnetic gauge group $S p\left(\widetilde{N}_{c}\right)$ with $N_{f}$ massive flavors [11, 15].

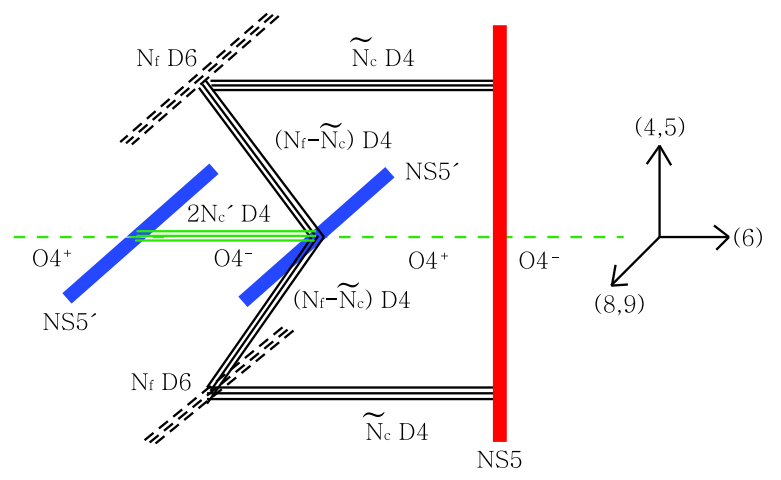

Figure 3: The nonsupersymmetric minimal energy brane configuration for the $S p\left(\widetilde{N}_{c}=\right.$ $\left.N_{f}+N_{c}^{\prime}-N_{c}-2\right) \times S O\left(2 N_{c}^{\prime}\right)$ gauge theory with $2 N_{f}$ quark fields $\widetilde{Q}$, a singlet field $\widetilde{X}$, the gauge singlet $\Phi, 2 N_{f}$ fields $N$, and an antisymmetric two index tensor $S$. For the M-theory lift, the position of these $N S 5_{L, R}^{\prime}$-branes are given by $v=m$ and those for the D4-branes is given by $v=2 m$ (its mirrors are given by $v=0$ ).

The type IIA and M-theory brane construction for the $\mathcal{N}=2$ gauge theory was described by [36] and after lifting the type IIA description we explained so far to M-theory, the corresponding magnetic M5-brane configuration with equal mass for the quarks where the gauge group is given by $S p\left(\widetilde{N}_{c}\right) \times S O\left(2 N_{c}^{\prime}\right)$ with fundamental matters for the first gauge group factor, in a background space of $x t=\prod_{k=1}^{N_{f}}\left(v^{2}-e_{k}^{2}\right)$ where this four dimensional space replaces 
(45610) directions, is characterized by

$$
t^{3}-\left(v^{2 \widetilde{N}_{c}+2}+m^{N_{f}}+\cdots\right) t^{2}+\left(v^{2 N_{c}^{\prime}}+\cdots\right) t-v^{2}\left[\prod_{k=1}^{N_{f}}\left(v^{2}-e_{k}^{2}\right)\right]=0
$$

where $e_{k}$ is the position of the D6-branes in the $v$ direction and we ignored the lower power terms in $v$ in $t^{2}$ and $t$ denoted by $\cdots$ and the scales for the gauge groups in front of the first term and the last term, for simplicity 10. When we consider the gauging of the maximal subgroup of the flavor symmetry group [6], the corresponding M5-brane configuration can be read off from this by putting the contribution from D6-branes(the coefficient of $v^{2}$ in the last term) to 1. Also the massless case in [7] can be obtained from this by taking the massless limit. Moreover, the case without D6-branes can be obtained from this curve by appropriate limit and will become (3.33) of [36]. If we take the limit where the last term in (3.1) vanishes, then the remaining piece becomes $\mathcal{N}=1 S p\left(\widetilde{N}_{c}\right)$ SQCD with $2 N_{c}^{\prime}$ matter fields [36, 37] and similarly for the limit where the first term of (3.1) will vanish, we obtain the remaining $\mathcal{N}=1$ $S O\left(2 N_{c}^{\prime}\right)$ SQCD with matter fields [36, 38].

From this curve (3.1) of cubic equation for $t$ above, the asymptotic regions can be classified by looking at the first two terms providing NS5-brane asymptotic region, next two terms providing $N S 5_{R}^{\prime}$-brane asymptotic region and the final two terms giving $N S 5_{L}^{\prime}$-brane asymptotic region as follows:

1. $v \rightarrow \infty$ limit implies

$$
w \rightarrow 0, \quad y \sim v^{2 \widetilde{N}_{c}+2}+\cdots \quad \text { NS asymptotic region. }
$$

2. $w \rightarrow \infty$ limit implies

$$
\begin{aligned}
& v \rightarrow m, \quad y \sim w^{2 N_{f}-2 N_{c}^{\prime}+2}+\cdots \quad N S_{L}^{\prime} \text { asymptotic region, } \\
& v \rightarrow m, \quad y \sim w^{2 N_{c}^{\prime}-2 \widetilde{N}_{c}-2}+\cdots \quad N S_{R}^{\prime} \text { asymptotic region. }
\end{aligned}
$$

As observed in [15], the two $N S 5_{L, R}^{\prime}$-branes are moving in the $v$ direction holding everything else fixed instead of moving D6-branes in the $v$ direction. Then the mirrors of D4-branes

\footnotetext{
${ }^{10}$ Of course, the corresponding supersymmetric electric M5-brane configuration from Figure 1 can be written similarly and the cubic equation can be written as follows: $t^{3}-\left(v^{2 N_{c}+2}+m^{N_{f}}+\cdots\right) t^{2}+\left(v^{2 N_{c}^{\prime}}+\right.$ $\cdots)\left[\prod_{k=1}^{N_{f}}\left(v^{2}-e_{k}^{2}\right)\right] t-v^{2}\left[\prod_{k=1}^{N_{f}}\left(v^{2}-e_{k}^{2}\right)\right]^{2}=0$ where $e_{k}$ is the position of the D6-branes in the $v$ direction and we put the equal mass $m$ for the quarks. The relevant part of 36 is explained in the subsection 4.4 where we need to specify when their function $J_{s} \equiv \prod_{k=i_{s-1}+1}^{i_{s}}\left(v^{2}-e_{k}^{2}\right)$ where $i_{k}$ is related to the number of D6-branes between the $(k-1)$-th and $k$-th NS5-brane by $d_{k}=i_{k}-i_{k-1}$ is nontrivial. In the present electric case, the D6-branes are located between NS5-brane and $N S 5_{R}^{\prime}$-brane from Figure 1 . It is easy to see that $J_{1}$ is a function of $v^{2}$ and their $J_{0}$ and $J_{2}$ are constant.
} 
are moved appropriately. In Figure 1, the origin of $v$ coordinate is located at the intersection of NS5-brane and D4-branes while in the coordinate we describe above $N S_{L, R}^{\prime}$ asymptotic region, the origin is located at the position of mirror D4-branes. Then the location of the original D6-branes is given by $v=2 \mathrm{~m}$. In other words, there is a shift in $v$ direction. The harmonic function sourced by the D6-branes can be written explicitly by summing of two contributions from two(original and its mirrors) $N_{f}$ D6-branes, as in [15]

$$
V(s)=1+\frac{N_{f} R}{\sqrt{f(s)^{2}+s^{2}}}+\frac{N_{f} R}{\sqrt{(f(s)-2 \Delta x)^{2}+s^{2}}} .
$$

Note that when we compare with the result of [12], the last term above is an extra contribution. For the straight line solution where $x^{4}=f(s)=\Delta x$, the differential equation for $g(s)$, which is equal to the magnitude of $w$, can be solved and it leads to

$$
g(s) \sim \exp \left[\frac{s}{4\left(N_{c}+1\right) R}\right]\left(\frac{s+\sqrt{(\Delta x)^{2}+s^{2}}}{R}\right)^{\frac{N_{f}}{2\left(N_{c}+1\right)}}
$$

and the $s$-independent integration constant can be fixed by the boundary condition from the above classification 2. From this solution, it is easy to see that even if $\Delta x$ goes to zero, the function $g(s)$ does not vanish. There is no smooth nonholomorphic M-theory curve. So the extra piece in the potential does not remove an instability from a new M5-brane mode.

\section{Conclusions and outlook}

In this paper, by gauging the maximal subgroup of the global flavor group for the model [4, 5], we have described the intersecting brane configuration of type IIA string theory in Figure 3 corresponding to the meta-stable nonsupersymmetric vacua of this gauge theory.

Also it is straightforward to take the dual gauge theory $S p\left(N_{c}\right) \times S O\left(2 \tilde{N}_{c}^{\prime}\right)$ and construct a meta-stable brane configuration in IIA string theory.

There exist many other examples of dynamical supersymmetry breaking in [39]-[46] and in [28, 29, 3, 47]. So it is natural to ask whether these examples(or their generalizations in many different directions) have the type IIA string theory brane configuration(if not, one should find out this brane configuration first) and it is an open problem to find out any new meta-stable brane configuration, along the lines of this paper if any. Some of the gauge theories can be described by type IIB string theory where there exist D5-branes, NS5-branes, NS5'-branes, D7-branes and O7-planes. We need to perform the Seiberg dual and add nonzero mass term for the quarks and sometimes the vanishing superpotential in the electric theory makes it easier frequently to deal with its nonvanishing dual magnetic superpotential. 


\section{Acknowledgments}

I would like to thank Y. Ookouchi for discussions on ISS model [2] and its relevant subjects. This work was supported by grant No. R01-2006-000-10965-0 from the Basic Research Program of the Korea Science \& Engineering Foundation. I thank KIAS(Korea Institute for Advanced Study) for hospitality where this work was undertaken.

\section{References}

[1] A. Giveon and D. Kutasov, Rev. Mod. Phys. 71, 983 (1999) [arXiv:hep-th/9802067].

[2] K. Intriligator, N. Seiberg and D. Shih, JHEP 0604, 021 (2006) arXiv:hep-th/0602239.

[3] K. Intriligator and N. Seiberg, arXiv:hep-ph/0702069.

[4] K. A. Intriligator and S. D. Thomas, Nucl. Phys. B 473, 121 (1996) arXiv:hep-th/9603158.

[5] K. I. Izawa and T. Yanagida, Prog. Theor. Phys. 95, 829 (1996) arXiv:hep-th/9602180].

[6] J. de Boer, K. Hori, H. Ooguri and Y. Oz, Nucl. Phys. B 522, 20 (1998) arXiv:hep-th/9801060.

[7] J. Hashiba, Nucl. Phys. B 550, 329 (1999) arXiv:hep-th/9809181].

[8] S. Forste, Phys. Lett. B 642, 142 (2006) arXiv:hep-th/0608036.

[9] C. Csaki, M. Schmaltz, W. Skiba and J. Terning, Phys. Rev. D 57, 7546 (1998) arXiv:hep-th/9801207.

[10] H. Ooguri and Y. Ookouchi, Phys. Lett. B 641, 323 (2006) arXiv:hep-th/0607183.

[11] S. Franco, I. Garcia-Etxebarria and A. M. Uranga, JHEP 0701, 085 (2007) arXiv:hep-th/0607218.

[12] I. Bena, E. Gorbatov, S. Hellerman, N. Seiberg and D. Shih, JHEP 0611, 088 (2006) arXiv:hep-th/0608157.

[13] C. Ahn, arXiv:hep-th/0702038.

[14] C. Ahn, arXiv:hep-th/0701145, to appear in JHEP.

[15] C. Ahn, Phys. Lett. B 647, 493 (2007) arXiv:hep-th/0610025]. 
[16] C. Ahn, Class. Quant. Grav. 24, 1359 (2007) arXiv:hep-th/0608160].

[17] K. A. Intriligator, R. G. Leigh and M. J. Strassler, Nucl. Phys. B 456, 567 (1995) arXiv:hep-th/9506148.

[18] H. Ooguri and Y. Ookouchi, Nucl. Phys. B 755, 239 (2006) arXiv:hep-th/0606061.

[19] N. J. Evans, C. V. Johnson and A. D. Shapere, Nucl. Phys. B 505, 251 (1997) arXiv:hep-th/9703210.

[20] J. H. Brodie and A. Hanany, Nucl. Phys. B 506, 157 (1997) arXiv:hep-th/9704043.

[21] I. Brunner, A. Hanany, A. Karch and D. Lust, Nucl. Phys. B 528, 197 (1998) arXiv:hep-th/9801017.

[22] J. L. F. Barbon, Phys. Lett. B 402, 59 (1997) arXiv:hep-th/9703051.

[23] R. Tatar, Phys. Lett. B 419, 99 (1998) [arXiv:hep-th/9704198].

[24] C. Ahn, K. Oh and R. Tatar, J. Geom. Phys. 31, 301 (1999) arXiv:hep-th/9707027].

[25] S. Elitzur, A. Giveon and D. Kutasov, Phys. Lett. B 400, 269 (1997) arXiv:hep-th/9702014.

[26] S. Elitzur, A. Giveon, D. Kutasov, E. Rabinovici and A. Schwimmer, Nucl. Phys. B 505, 202 (1997) arXiv:hep-th/9704104.

[27] A. Hanany and E. Witten, Nucl. Phys. B 492, 152 (1997) arXiv:hep-th/9611230.

[28] J. Terning, "Modern supersymmetry: Dynamics and duality," Oxford, UK: Clarendon (2006) $324 p$

[29] J. Terning, arXiv:hep-th/0306119.

[30] K. A. Intriligator and P. Pouliot, Phys. Lett. B 353, 471 (1995) arXiv:hep-th/9505006.

[31] K. Hori, Nucl. Phys. B 540, 187 (1999) arXiv:hep-th/9805142.

[32] D. Shih, arXiv:hep-th/0703196.

[33] Z. Chacko, M. A. Luty and E. Ponton, JHEP 9812, 016 (1998) arXiv:hep-th/9810253.

[34] L. O'Raifeartaigh, Nucl. Phys. B 96, 331 (1975). 
[35] H. Murayama and Y. Nomura, arXiv:hep-ph/0701231.

[36] K. Landsteiner, E. Lopez and D. A. Lowe, Nucl. Phys. B 507, 197 (1997) arXiv:hep-th/9705199.

[37] C. Ahn, K. Oh and R. Tatar, Phys. Rev. D 58, 086002 (1998) arXiv:hep-th/9708127.

[38] C. Ahn, K. Oh and R. Tatar, Phys. Lett. B 416, 75 (1998) arXiv:hep-th/9709096.

[39] I. Affleck, M. Dine and N. Seiberg, Phys. Rev. Lett. 52, 1677 (1984).

[40] I. Affleck, M. Dine and N. Seiberg, Nucl. Phys. B 256, 557 (1985).

[41] H. Murayama, Phys. Lett. B 355, 187 (1995) arXiv:hep-th/9505082.

[42] E. Poppitz, Y. Shadmi and S. P. Trivedi, Phys. Lett. B 388, 561 (1996) arXiv:hep-th/9606184.

[43] C. Csaki, L. Randall, W. Skiba and R. G. Leigh, Phys. Lett. B 387, 791 (1996) arXiv:hep-th/9607021.

[44] K. A. Intriligator and S. D. Thomas, arXiv:hep-th/9608046].

[45] S. Dimopoulos, G. R. Dvali, R. Rattazzi and G. F. Giudice, Nucl. Phys. B 510, 12 (1998) arXiv:hep-ph/9705307.

[46] M. A. Luty and J. Terning, Phys. Rev. D 57, 6799 (1998) arXiv:hep-ph/9709306.

[47] Y. Shadmi and Y. Shirman, Rev. Mod. Phys. 72, 25 (2000) arXiv:hep-th/9907225. 